\title{
Tracking key virulence loci encoding aerobactin and salmochelin siderophore synthesis in Klebsiella pneumoniae
}

\author{
Margaret M. C. Lam¹, Kelly L. Wyres', Louise M. Judd', Ryan R. Wick', Adam Jenney ${ }^{3}$, Sylvain Brisse ${ }^{2}$
} and Kathryn E. Holt ${ }^{1,4^{*}}$

\begin{abstract}
Background: Klebsiella pneumoniae is a recognised agent of multidrug-resistant (MDR) healthcare-associated infections; however, individual strains vary in their virulence potential due to the presence of mobile accessory genes. In particular, gene clusters encoding the biosynthesis of siderophores aerobactin (iuc) and salmochelin (iro) are associated with invasive disease and are common amongst hypervirulent $K$. pneumoniae clones that cause severe community-associated infections such as liver abscess and pneumonia. Concerningly, iuc has also been reported in MDR strains in the hospital setting, where it was associated with increased mortality, highlighting the need to understand, detect and track the mobility of these virulence loci in the K. pneumoniae population.
\end{abstract}

Methods: Here, we examined the genetic diversity, distribution and mobilisation of iuc and iro loci amongst 2503 K. pneumoniae genomes using comparative genomics approaches and developed tools for tracking them via genomic surveillance.

Results: Iro and iuc were detected at low prevalence $(<10 \%)$. Considerable genetic diversity was observed, resolving into five iro and six iuc lineages that show distinct patterns of mobilisation and dissemination in the K. pneumoniae population. The major burden of iuc and iro amongst the genomes analysed was due to two linked lineages (iuc1/iro1 74\% and iuc2/iro2 14\%), each carried by a distinct non-self-transmissible IncFIB $\mathrm{B}_{\mathrm{K}}$ virulence plasmid type that we designate KpVP-1 and KpVP-2. These dominant types also carry hypermucoidy ( $r m p A)$ determinants and include all previously described virulence plasmids of $K$. pneumoniae. The other iuc and iro lineages were associated with diverse plasmids, including some carrying IncFll conjugative transfer regions and some imported from Escherichia coli; the exceptions were iro3 (mobilised by ICEKP1) and iuc4 (fixed in the chromosome of K. pneumoniae subspecies rhinoscleromatis). Iro/iuc mobile genetic elements (MGEs) appear to be stably maintained at high frequency within known hypervirulent strains (ST23, ST86, etc.) but were also detected at low prevalence in others such as MDR strain ST258.

Conclusions: IUC and iro are mobilised in K. pneumoniae via a limited number of MGEs. This study provides a framework for identifying and tracking these important virulence loci, which will be important for genomic surveillance efforts including monitoring for the emergence of hypervirulent MDR K. pneumoniae strains.

Keywords: Klebsiella pneumoniae, Virulence, Hypervirulence, Salmochelin, Aerobactin, Virulence plasmids, Plasmids, Invasive disease, Genomic surveillance

\footnotetext{
* Correspondence: kat.holt@lshtm.ac.uk

${ }^{1}$ Department of Biochemistry and Molecular Biology, Bio21 Molecular Science

and Biotechnology Institute, University of Melbourne, Parkville, Victoria 3010,

Australia

${ }^{4}$ London School of Hygiene \& Tropical Medicine, London WC1E 7HT, UK

Full list of author information is available at the end of the article
}

(c) The Author(s). 2018 Open Access This article is distributed under the terms of the Creative Commons Attribution 4.0 International License (http://creativecommons.org/licenses/by/4.0/), which permits unrestricted use, distribution, and reproduction in any medium, provided you give appropriate credit to the original author(s) and the source, provide a link to the Creative Commons license, and indicate if changes were made. The Creative Commons Public Domain Dedication waiver (http://creativecommons.org/publicdomain/zero/1.0/) applies to the data made available in this article, unless otherwise stated. 


\section{Background}

The enteric opportunistic bacterial pathogen Klebsiella pneumoniae imposes an increasing infection burden worldwide $[1,2]$. These infections typically fall into one of two distinct categories: healthcare-associated (HA) infections caused by strains that are frequently multidrug-resistant (MDR) and community-associated (CA) infections arising from the so-called hypervirulent strains that can cause highly invasive infections such as liver abscess but are usually drug sensitive $[2,3]$. The antimicrobial resistance (AMR) and/or virulence determinants possessed by the associated bacteria are generally found on mobile genetic elements (MGEs) that transmit between K. pneumoniae cells via horizontal gene transfer (HGT) [4]. These MGEs, most typically plasmids and integrative and conjugative elements (ICEs), are therefore important constituents of the accessory genome that imbue $K$. pneumoniae organisms with their distinct HA or CA clinical profiles.

It is apparent that a wide diversity of $K$. pneumoniae can cause infections in hospitalised patients $[3,5,6]$ and that basic pathogenicity factors such as lipopolysaccharide, capsular polysaccharide, type 3 fimbriae and the siderophore enterobactin (Ent) are common to all K. pneumoniae and conserved in the chromosome as core genes [1, 3]. However, enhanced virulence or 'hypervirulence' is associated with specific capsular serotypes (K1, K2, K5) and with MGE-encoded accessory genes that are much rarer in the K. pneumoniae population [3]. Of particular importance are those encoding additional siderophore systems, namely yersiniabactin (Ybt) $[3,7,8]$, aerobactin (Iuc) [9] and salmochelin (Iro) [10].

Synthesis of acquired siderophores contributes to $K$. pneumoniae virulence via multiple mechanisms. However, iron assimilation via the conserved siderophore Ent is hampered by human neutrophils and epithelial cells through the secretion of lipocalin-2 (Lcn2), which binds, and thus inhibits bacterial uptake of, iron-loaded Ent [11]. Ybt, Iro and Iuc on the other hand are not subject to Lcn2 binding; Iro is a glycosylated derivative of Ent, while Ybt and Iuc possess an entirely distinct structure from Ent. The ability of salmochelin to counter Lcn2 binding is important for bacterial growth and has been shown to correlate with enhanced virulence in a mouse sepsis model [12]. The association between aerobactin and virulence has long been recognised, with multiple studies demonstrating its key role in an increased iron acquisition, bacterial growth and/or virulence in various murine models, human ascites fluid and blood [9, 13-15]. Even in strains that possess all four siderophore-encoding loci, Iuc appears to play the most critical role in virulence both in vitro and in vivo [13] and serves as an important biomarker for identifying hypervirulent isolates [16].

In K. pneumoniae, Ybt biosynthesis is encoded by the $y b t$ locus, which is typically located on a chromosomal
ICE known as ICEKp (of which there are at least 14 distinct variants) and was recently also reported on plasmids [7, 8, 17]. A screen of $2500 \mathrm{~K}$. pneumoniae genomes showed $y b t$ to be prevalent in one third of the sequenced population and associated with hundreds of putative ICEK $p$ acquisition events across the chromosomes of both hypervirulent and MDR lineages [8]. In contrast, Iuc and Iro synthesis is encoded by loci (iuc and iro, depicted in Fig. 1), that are typically co-located on the so-called 'virulence plasmids' of K. pneumoniae. The best characterised virulence plasmids are the $224 \mathrm{kbp}$ plasmid $\mathrm{pK} 2044$ from serotype $\mathrm{K} 1$, sequence type (ST) 23 strain NTUH-K2044 [18]; the $219 \mathrm{kbp}$ plasmid pLVPK from K2, ST86 strain CG43 [19]; and the $121 \mathrm{kbp}$ plasmid Kp52.145pII from serotype K2, ST66 strain Kp52.145 (strain also known as 52145 or B5055; plasmid also known as pKP100) [9, 20]. These plasmids also carry additional virulence determinants including rmpA genes that upregulate capsule production, conferring a hypermucoid phenotype that is considered a hallmark of hypervirulent strains [21], other gene clusters associated with iron uptake and utilisation and other loci encoding resistance to heavy metals such as copper (pco-pbr), silver (sil) and tellurite (ter) [4]. In addition to the virulence plasmid-encoded iro and rmpA genes, the ST23 strain NTUH-K2044 also carries a chromosomal copy of iro and rmpA located within ICEKp1 [7]; however, this is not a typical feature of ST23 [22].

The majority of $K$. pneumoniae lineages associated with liver abscess and other invasive community-acquired infections (e.g. clonal group (CG) 23, CG86, CG380) carry virulence plasmids encoding iro, iuc and $r m p A[3,9,16,23-25]$. However, while virulence and AMR genes are both transmitted within the $K$. pneumoniae population via plasmids, until recently, these plasmids have mainly been segregated in non-overlapping populations such that the virulence plasmids encoding iuc and iro have rarely been detected in MDR populations that cause HA infections and outbreaks [3, 4, 26]. However, the virulence plasmid Kp52.145pII has been shown experimentally to be mobilisable [21], and there are emerging reports of MDR clones such as ST11, ST147 and ST15 acquiring virulence plasmids $[27,28]$. The combination of hypervirulence and MDR can result in invasive infections that are very difficult to treat. This can result in dangerous hospital outbreaks; for example, an aerobactin-producing carbapenemase-producing ST11 strain recently caused a fatal outbreak of ventilator-associated pneumonia in a Chinese intensive care unit, with $100 \%$ mortality $[27,29]$. AMR plasmids are also occasionally acquired by ST23 and other hypervirulent $K$. pneumoniae clones [25, 30, 31].

The ease with which virulence plasmids spread in the $K$. pneumoniae population poses a significant global health threat, highlighting the importance of understanding and 


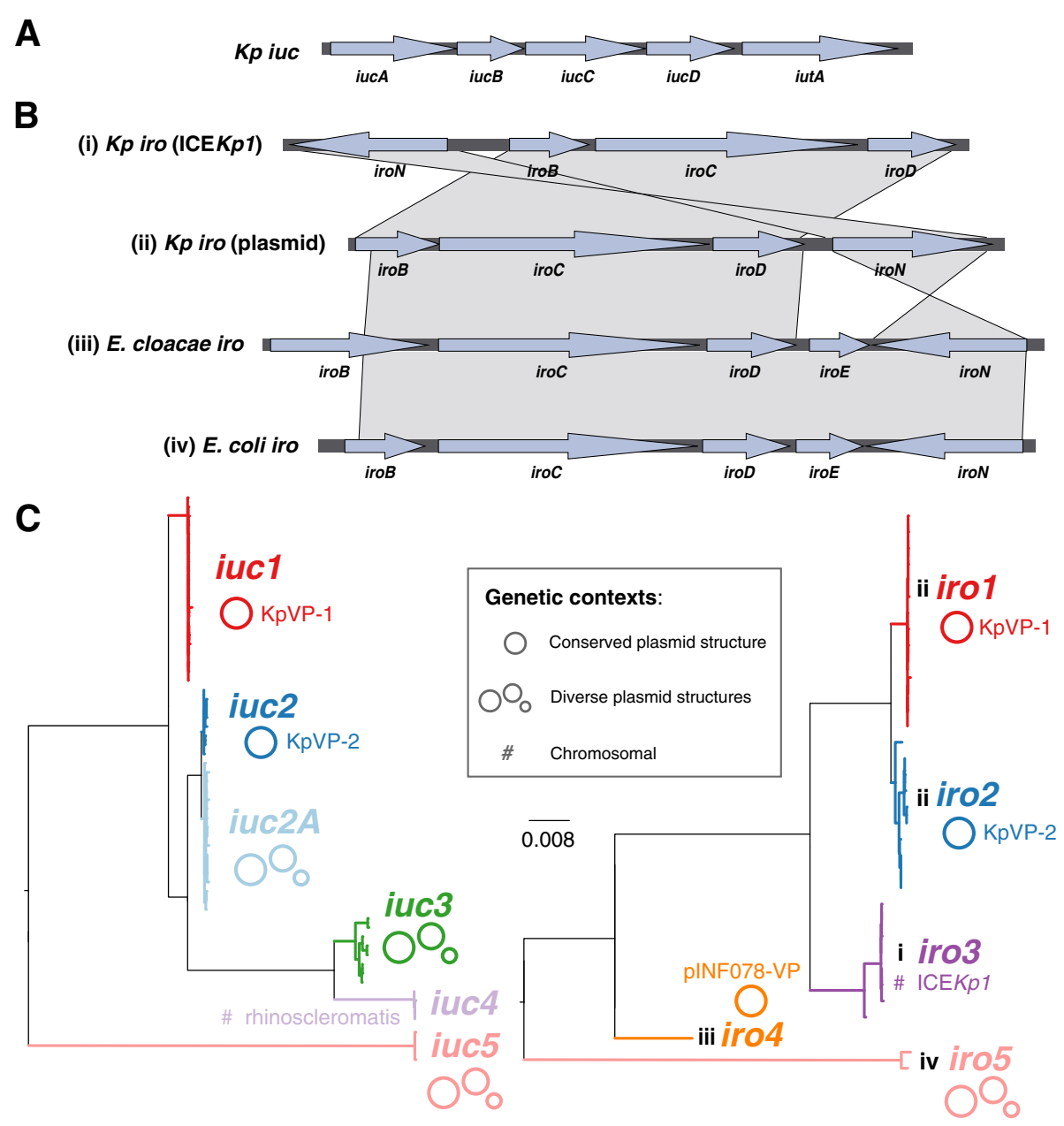

Fig. 1 Aerobactin and salmochelin locus variants found in Klebsiella pnuemoniae. a A single aerobactin (iuc) locus structure was found in $K$. pneumoniae. b Four different structures of the salmochelin (iro) locus were found in K. pneumoniae (i-iv). Note two of these are typical of structures found in other species (iii in Enterobacter cloacae, iv in Escherichia coli). c Maximum likelihood phylogenetic trees inferred from iuc and iro sequence types (AbSTs and SmSTs) identified in K. pneumoniae genomes. Phylogenetic lineages discussed in the text are labelled and their mobility indicated; nucleotide divergence within and between lineages is given in Additional files 8 and 9. Iro locus structures associated with each lineage are labelled $\mathrm{i}-\mathrm{iv}$, as defined in panel $\mathbf{b}$

monitoring the movement of these loci between different strains and clones. Here, we investigate the diversity of aerobactin and salmochelin synthesis loci in $2733 \mathrm{~K}$. pneumoniae complex genomes, aiming to understand the diversity and distribution of these virulence loci in the population and to develop a framework for their inclusion in genomic surveillance efforts.

\section{Methods}

\section{Bacterial genome sequences}

2733 genomes of the K. pneumoniae complex, including isolates collected from diverse sources and geographical locations, were analysed in this study (see Additional file 1). The genomes represent a convenience sample of our own isolate collections from clinical and species diversity studies $[5,8,22,32]$, as well as sequences that were publicly available in GenBank or via the NCTC 3000 project (https://www.sanger.ac.uk/resources/downloads/bacteria/ nctc/) at the commencement of the study (June 2017). The majority of these genomes were also included in our previous genome study screening for yersiniabactin and colibactin [8].

For $n=1847$ genomes (see Additional file 1), Illumina short reads were available, and these were used to generate consistently optimised de novo assembly graphs using Unicycler v0.3.0b with SPAdes v3.8.1 [33, 34]. The remaining $n=886$ genomes were publicly available only in the form of draft genome assemblies, i.e. with no reads available for direct analysis. All genome assemblies were re-annotated using Prokka [35] to allow for standardised comparison. All genomes were assigned to species by comparison to a curated set of Enterobacteriaceae genomes 
using mash (implemented in Kleborate, https://github.com/ katholt/Kleborate); this confirmed $2503 \mathrm{~K}$. pneumoniae, 12 Klebsiella quasipneumoniae subsp. quasipneumoniae, $59 \mathrm{~K}$. quasipneumoniae subsp. similipneumoniae, 158 Klebsiella variicola and 1 Klebsiella quasivariicola (Additional file 1).

\section{Long-read sequencing of isolates}

Three isolates in our own collection (INF078, INF151, INF237) carried novel iuc and/or iro plasmids identified from short-read Illumina data. We subjected these to long-read sequencing using a MinION R9.4 flow cell (Oxford Nanopore Technologies (ONT)) device in order to resolve the complete sequences for the relevant plasmids. Overnight cultures of each isolate were prepared in LB broth at $37{ }^{\circ} \mathrm{C}$, and DNA extracted using Agencourt Genfind v2 (Beckman Coulter) according to a previously described protocol (doi: https://doi.org/10.17504/ protocols.io.p5mdq46). Sequencing libraries were prepared using a 1D ligation library (SQK-LSK108) and native barcoding (EXP-NBD103) as previously described [22, 36]. The resulting reads were combined with their respective Illumina reads to generate a hybrid assembly using our Unicycler software v0.4.4-beta [33, 36]. Note this approach uses ONT reads to bridge together contig sequences constructed from Illumina data, followed by consensus base call polishing with both types of reads. Annotations for the hybrid assemblies were generated as described above, and the annotated sequences submitted to GenBank under accession numbers QWFT01000001-QWFT01000009, and CP032831-CP032838 (Additional files 1, 2 and 3).

\section{Multi-locus sequence typing}

Chromosomal sequence types were determined for each genome assembly using the BIGSdb- $K p$ seven-locus multilocus sequence typing (MLST) scheme [37] screened using Kleborate (https://github.com/katholt/Kleborate). A novel ST (ST3370) was identified and added to the BIGSdb-Kp MLST database.

To facilitate the development of MLST schemes for the aerobactin and salmochelin biosynthesis loci $i u c$ and iro, alleles for genes belonging to each locus (i.e. iuc $A B C D$, iutA; and iroBCDN; respectively) from genomes with 'typeable' loci (defined as those in which all genes in the locus had high-quality consensus base calls when mapping with SRST2) were extracted by comparison to known alleles in the BIGSdb-Kp virulence database (http://bigsdb. pasteur.fr/klebsiella/klebsiella.html) [25], using SRST2 v0.2.0 [38] to screen Illumina read sets where available and BLAST+ v2.2.30 to screen assemblies. Incomplete, 'non-typeable' iro and iuc loci were excluded from the MLST scheme (marked NT in Additional file 1). Each unique combination of alleles was assigned an aerobactin sequence type (AbST) or salmochelin sequence type (SmST), defined in Additional files 4 and 5. The AbST and SmST schemes, profiles and corresponding alleles are also available in the BIGSdb- $K p$ database and in the Kleborate Github repository (see links above).

\section{Identification of other genes of interest and genetic context of iuc and iro loci}

Capsule (K) loci were identified in each assembled genome using Kaptive [39]. RmpA gene copy number was determined by BLASTn search of all genome assemblies using the rmpA and rmpA2 sequences from pK2044 (GenBank accession AP006726.1) as queries with $>90 \%$ coverage and $>90 \%$ nucleotide identity. Similarly, BLASTn was used to screen the genome assemblies for the $\operatorname{IncFIB}_{K}$ repA sequence from virulence plasmids $\mathrm{pK} 2044$ and Kp52.145 pII (GenBank accession FO834905.1), with IncFIB $_{K}$ presence defined as $>90 \%$ coverage and $>80 \%$ nucleotide identity to these query sequences (to ensure inclusion of known $\operatorname{IncFIB}_{\mathrm{K}}$ sequences while excluding detection of non- FIB $_{K}$ sequences such as the IncFIB sequences frequently detected in other Enterobacteriaceae bacteria). IncFII replicons were identified using BLASTn search of the PlasmidFinder database [40].

Assemblies of all iuc+ or iro+ genomes were manually inspected to determine whether the loci of interest were located on the chromosome or on previously described virulence plasmids (pK2044 and Kp52.145pII). This confirmed most to be located in the chromosome (iro3 in ICEKp1 or iuc4 in the subspecies rhinoscleromatis lineage) or one of the known plasmids. For the remaining genomes, annotated contigs containing the $i u c$ and/or iro loci were checked for known chromosomal or plasmid features, aided by BLASTn searching against the NCBI non-redundant nucleotide database and inspection of the assembly graphs using Bandage v0.8.0 [41].

\section{Phylogenetic analyses}

Maximum likelihood phylogenetic trees capturing the relationships between AbSTs or SmSTs were constructed by aligning the allele nucleotide sequences corresponding to each sequence type within each scheme using MUSCLE v3.8.31 [42] then using each of the two alignments (one for AbSTs, one for SmSTs) as input for phylogenetic inference in RAxML v7.7.2 [43]. For each alignment, RAxML was run five times with the generalised time-reversible model and a gamma distribution, and the trees with the highest likelihood were selected. Lineages were defined as monophyletic groups of AbSTs or SmSTs, which were each associated with a unique MGE structure; STs within lineages shared $\geq 2$ alleles (for SmSTs) or $\geq 3$ alleles (for AbST), whereas no alleles were shared between lineages.

Maximum likelihood phylogenies were similarly constructed for (i) aerobactin and salmochelin locus alignments populated by sequences extracted from BLAST 
hits amongst representatives of the wider Enterobacterales order (representatives listed in Additional file 6) and (ii) IncFIB $_{K}$ replicon sequence alignments constructed by mapping iuc-positive (iuc+) and iro-positive (iro+) genomes to a reference IncFIB $_{\mathrm{K}}$ sequence (coordinates 128130 to 132007 , spanning repA to $\operatorname{sop} B$, of the pK2044 plasmid sequence; GenBank accession AP006726.1).

\section{Plasmid comparisons}

Twelve representative plasmids (10 complete, including $n=3$ generated from hybrid long- and short-read assemblies detailed above, and 2 partial) were chosen for comparative analysis (these are available as a set in FigShare under doi: https://doi.org/10.6084/m9.figshare.6839981; and see Additional file 2 for list of sources and GenBank accession numbers). Six of these representative plasmids were sourced from the NCTC 3000 project (https:// www.sanger.ac.uk/resources/downloads/bacteria/nctc/).

As no complete plasmid sequences from $K$. pneumoniae were available with iuc5, we used plasmid p3PCN033 from E. coli as the reference for iuc5. We consider this appropriate in the circumstances since the K. pneumoniae iuc5 plasmids shared with p3PCN033 the IncFII replicon (native to E. coli) and the iuc and iro sequences and structural variants typical of E. coli; the iuc5 contigs from K. pneumoniae showed 99.19-99.95\% sequence identity with p3PCN033, and IncFII plasmids while considered native to $E$. coli have been detected in Klebsiella pneumoniae alongside other Enterobacteriaceae members [44, 45].

The representative plasmid sequences were compared using Mauve v2.4.0 [46], in order to identify homology blocks conserved amongst subsets of the plasmids. BLASTn comparisons of related plasmids were plotted using GenoPlotR v0.8.7 package [47] for R. All iuc+ or iro+ genomes were mapped against all 12 representative plasmids in order to calculate the coverage of each plasmid in each genome. This was done using Bowtie2 v2.2.9 [48] to map Illumina reads where available, and $100 \mathrm{bp}$ reads simulated from draft assemblies where raw sequence reads were not available, using the RedDog pipeline (https://github.com/katholt/RedDog). For every gene annotated within each reference plasmid, the proportion of isolates within each group of genomes sharing the same iuc/iro lineage carrying the gene was calculated using the gene presence/absence table reported by RedDog (presence defined as $\geq 95 \%$ of the length of the gene being covered by at least five reads) and plotted as circular heatmaps using ggplot2 in $\mathrm{R}$ (using geom_tile to achieve a heatmap grid and polar_coord to circularise).

\section{Results}

Prevalence of iuc and iro in K. pneumoniae

Iuc and iro were detected only in K. pneumoniae genomes, and not in other members of the K. pneumoniae species complex. Of the $2503 K$. pneumoniae genomes screened, iuc was detected in $8.7 \%(n=217)$ and iro in $7.2 \%$ ( $n=181$; listed in Additional file 1, excluding those with a partial iro locus as discussed below). The presence of intact iro and iuc loci was strongly associated (odds ratio (OR) 711, 95\% confidence interval (CI) 386-1458, $p<1 \times$ $\left.10^{-16}\right)$, co-occurring in 162 genomes $(6.5 \%$ of the genomes tested). The iro locus appears to be susceptible to deletion; partial iro loci were observed in $n=50 \mathrm{~K}$. pneumoniae isolates (noted as iro in Additional file 1), mostly those that were isolated from historical collections prior to 1960. Of 39 isolates collected prior to 1960 and with any iro genes present, 36 (92\%) carried deletion variants of the locus, compared to $4 / 163(2.5 \%)$ amongst isolates from 1975 onwards (OR 416, 95\% CI 88-3297, $p<2 \times 10^{-16}$ ). As expected, the presence of iuc and iro was each strongly associated with the presence of $\operatorname{rmpA}$, with 157 genomes carrying all three loci (excluding partial iro). A total of 238 genomes (9.5\%) carried rmpA genes: $n=110$ (4.4\%) carried one, $n=127$ (5.1\%) carried two, and a single genome, ST23 NTUH-K2044, carried three (as described previously [7, 18], see Additional file 1).

\section{Genetic diversity of iuc and iro in K. pneumoniae}

Next, we explored nucleotide diversity of the genes comprising the iro and iuc loci in K. pneumoniae. The five genes comprising the iuc locus (Fig. 1a) and four genes of the K. pneumoniae forms of the iro locus (Fig. 1b) were screened for sequence variation, and each unique gene sequence variant was assigned an allele number. Of the $n=$ 209 genomes carrying a typeable iuc locus, 62 unique iuc allele combinations were observed and assigned a unique aerobactin sequence type or AbST (see Additional file 4 for AbST definitions and Additional file 1 for AbSTs assigned to each genome). The iutA alleles present in the iuc locus showed $>28 \%$ nucleotide divergence from a core chromosomal paralog of iutA encoding a TonB-dependent siderophore receptor (positions 2043670-2045871 in NTUH-K2044), which we observed in $96.4 \%$ of all genomes; the alleles of this core chromosomal gene are not included in the aerobactin MLST scheme. Typeable iro loci were identified in $n=164$ genomes, comprising 35 unique salmochelin sequence types or SmSTs (defined in Additional file 5, see Additional file 1 for SmSTs assigned to each genome). Maximum likelihood phylogenetic analyses of the AbST and SmST sequences, and their translated amino acid sequences, revealed five highly distinct iuc lineages and five iro lineages (labelled iro1, iro2 etc.; see Fig. 1c, Additional file 7). Nucleotide divergence between lineages was 1-11\% (20-1000 substitutions), and no alleles were shared between lineages (Additional files 8 and 9). Nucleotide divergence within lineages was low, with mean divergence of $0.001-0.40 \%$ (iro) and 0.013-0.50\% (iuc) (Additional files 8 and 9) and 
at least two (iro) or three (iuc) shared alleles between members of the same lineage. Of note, the iro4, iro5 and iuc5 loci were quite distant from other lineages (each showing $>5.5 \%$ nucleotide divergence from all other lineages vs $<4.6 \%$ divergence amongst the other lineages; Fig. 1, Additional files 8 and 9). Comparison to iuc and iro genes present in other bacteria (all of which were members of the order Enterobacterales, see Additional files 10 and 11), and the presence of the additional $i r o E$ gene that we observed in other bacteria (all of which were members of family Enterobacteriaceae, see Fig. 1b), suggests that these more distant lineages derive from outside Klebsiella, most likely Enterobacter (iro4) and E. coli (iro5, iuc5). Note that genotyping of $r m p A$ was not performed since most $r m p A$ -positive genomes carry two copies of the gene, which complicates allele typing from short-read data; however, $r m p A$ copy number per genome is reported in Additional file 1.

\section{Mobile genetic elements associated with iuc and iro loci} Inspection of the genetic context surrounding the iuc and iro sequences revealed that the various iuc and iro lineages were associated with distinct MGEs, with the exception of iuc4 which was restricted to the chromosome of $K$. pneumoniae subspecies rhinoscleromatis (ST67) (Fig. 1c, Table 1). Most common were iuc1 and iro1; these were both associated with pK2044-like plasmids (hereafter called KpVP1-1, see below) and the presence of two rmpA genes and accounted for $74 \%$ of all iuc+iro+ genomes. These were followed by $i u c 2$ and iro2, which were associated with Kp52.145 pII-like plasmids (hereafter called KpVP-2, see below), the presence of one rmpA gene, and accounted for $14 \%$ of all iuc+iro + genomes. A sister clade of $i u c 2$, which we named $i u c 2 a$, was associated with diverse plasmids that shared some homology with Kp52.145 pII (36-70\% coverage,
99\% nucleotide identity). Most iuc $2 a+$ isolates carried a single rmpA gene $(n=38,88.4 \%)$, and all lacked an intact iro locus ( $n=26,60.5 \%$ had a partial iro locus). Lineage iuc3 was related to the iuc4 lineage encoded on the rhinoscleromatis chromosome but was present on novel plasmids. Iro3 was located within the chromosomally integrated ICEKp1, along with rmpA. Four genomes carried iuc5 (two of these also carried iro5; all lacked rmpA). The iuc5 sequences were distantly related to iuc 1 and iuc2 (> 8.9\% nucleotide divergence) but were identical to sequences found in E. coli and located on contigs that matched closely to E. coli AMR plasmids (e.g. strain PCN033 plasmid p3PCN033, accession CP006635.1 [49], which showed $>99 \%$ nucleotide identity to the best assembled of iuc5+ K. pneumoniae contigs). Iro4 was identified in a single genome (which lacked $r m p A$ ) and was $>6.1 \%$ divergent from iro 1 and iro 2 sequences. Its closest known relatives are iro sequences present in the chromosomes of Enterobacter cloacae and Enterobacter hormaechei (strains AR_0065, accession CP020053.1, and 34977, accession CP010376.2, respectively; 95\% identity). Lineages iro4 and iro5 follow the gene configuration typical of non- $K$. pneumoniae Enterobacteriaceae iro loci, from which the $K$. pneumoniae iro1, iro2 and iro3 differ by lack of iroE and inversion of iroN (see Fig. 1b).

To examine the gene content and replicon differences between the various $K$. pneumoniae plasmids associated with $i u c$ and/or iro, 12 representative plasmids associated with the various lineages were selected for comparison (Fig. 2, Additional file 2). These include six complete $K$. pneumoniae plasmid sequences identified from finished genomes: iuc1/iro1 $(n=1)$, iuc2/iro2 $(n=1), i u c 2 a(n=3)$, iuc3 $(n=1)$; three novel complete K. pneumoniae plasmid sequences that we generated for this study, carrying iuc $2 a$ $(n=2)$ and iro4 $(n=1)$; and two large contigs that we

Table 1 Summary of iuc and/or iro plasmid lineages

\begin{tabular}{|c|c|c|c|}
\hline Lineage(s) & $N$ & Mobile genetic element & Reference(s) \\
\hline iucl $(+$ irol) & $121(119)$ & $\begin{array}{l}\text { K. pneumoniae VP-1, type I IncFIB } \mathrm{K}+\operatorname{IncHI1B} \text {, } \\
\text { rmpA+rmpA2 }\end{array}$ & pK2044 (accession AP006726.1) \\
\hline iuc2 (+ iro2) & $23(23)$ & K. pneumoniae VP-2, type II IncFIB ${ }_{K}, r m p A$ & Kp52.145 plasmid II (accession FO384905.1) \\
\hline iuc2a & 43 & $\begin{array}{l}\text { Novel, diverse plasmids } \\
\text { IncFIB }_{K}+\text { other IncF replicons, sometimes IncFll tra }\end{array}$ & $\begin{array}{l}\text { Many distinct types } \\
\text { Novel examples: pINF151_01-VP (accession QWFT01000004), } \\
\text { pINF237_01-VP (accession CP032834) }\end{array}$ \\
\hline iuc3 & 11 & $\begin{array}{l}\text { Novel, diverse plasmids } \\
\text { IncFIB } B_{K}+\text { IncFII tra }\end{array}$ & NCTC11676, NCTC11697 \\
\hline iuc4 & 7 & Chromosomal integration & $\begin{array}{l}\text { K. pneumoniae rhinoscleromatis, e.g. strain SB3432 } \\
\text { (accession FO203501.1) }\end{array}$ \\
\hline iuc5 (+iro5) & $4(2)$ & $\begin{array}{l}\text { E. coli IncFll tra plasmid } \\
\text { E. coli iroBCDEN + AMR }\end{array}$ & E. coli strain PCN033 plasmid p3PCN033 (accession CP006635.1) \\
\hline iro3 & 16 & Chromosomal ICEKpl & K. pneumoniae NTUH-K2044 ICEKp1 (accession AB298504.1) \\
\hline iro4 & 1 & $\begin{array}{l}\text { Novel plasmid } \\
\text { IncFIB }+ \text { IncFIl tra } \\
\text { E. cloacae/E. hormaechei iroBCDEN (× } 13 \text { copies })\end{array}$ & pINF078-VP (accession CP032832) \\
\hline
\end{tabular}




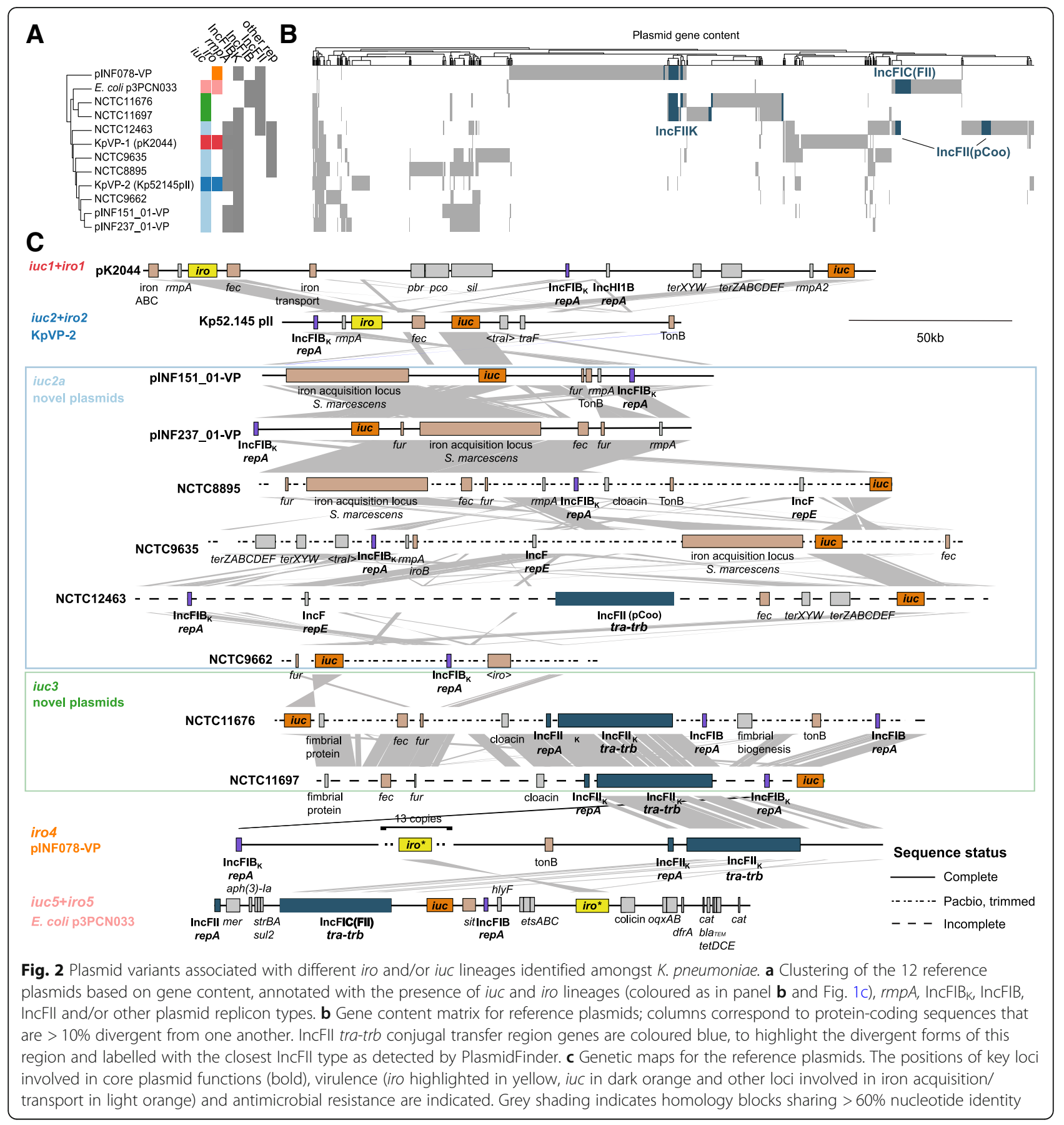

identified from public K. pneumoniae genome data representing partial sequences for additional plasmids carrying iuc2a $(n=1)$ and iuc3 $(n=1)$ (Fig. 2). The K. pneumoniae genomes in which iuc5/iro5 were identified were available only as draft assemblies deposited in public databases, and the associated plasmid sequences were fragmented in these assemblies; hence, we used E. coli strain PCN033 plasmid p3PCN033 [49] as the representative for iuc5/ iro5. The representative plasmid sequences differed substantially in their structure and gene content between and within the different lineages (Fig. 2b, c).

All representative iuc or iro plasmids harboured an $\operatorname{IncFIB}_{\mathrm{K}}(n=9)$ or IncFIB $(n=3)$ replicon, including the repA replication gene, oriT origin of transfer and sop $A B$ partitioning genes (presence of these replicons in each plasmid is indicated purple in Fig. 2c and listed in Additional file 2). The $\operatorname{IncFIB}_{\mathrm{K}}$ replicon was present in $n=$ 202/208 (97\%) of isolates with plasmid-encoded iuc or iro, 
including $100 \%$ of iuc1/iro1, iuc2/iro2, iuc $2 a$ and iro4 isolates, and $82 \%$ of $i u c 3$ isolates. Each of these iuc/iro lineages was associated with a unique sequence variant of the IncFIB $_{\mathrm{K}}$ replicon (see tree in Fig. 3 and nucleotide identity with the $\mathrm{IncFIB}_{\mathrm{K}}$ rep sequences from KpVP-1 and KpVP-2 listed in Additional file 1), supporting the segregation of the iuc and iro loci with distinct $\mathrm{FIB}_{\mathrm{K}}$ plasmid backbones. However, the $\mathrm{IncFIB}_{\mathrm{K}}$ replicon was also widely detected amongst isolates that do not carry iro and iuc (77\% of all K. pneumoniae genomes and $69 \%$ amongst other species in the complex; see Additional file 1), including MDR K. pneumoniae lineages such as CG258, and is known to be associated with AMR plasmids $[44,50]$. IncFIB replicons, which are common amongst $E$. coli and display $>39 \%$ nucleotide divergence from the $\mathrm{IncFIB}_{\mathrm{K}}$ replicon, were found in all $K$. pneumoniae isolates carrying the E. coli variant iuc5 (100\%) and also detected in two isolates carrying iuc3 plasmids (18\%; marked in Fig. 2a, c), suggesting the transfer of these $i u c$ variants into K. pneumoniae via such plasmids.

In order to explore structural conservation of plasmids amongst isolates with each iro or iuc lineage, we mapped the sequence data from all isolates carrying either of these loci against the 12 representative plasmid sequences (Fig. 4). This revealed that plasmid structures were largely conserved amongst isolates sharing the same iuc or iro lineages, although plasmids associated with $i u c 2 a$ and iuc 3 showed more diversity than others (Fig. 4 and see below). The distribution of iuc and iro variants with respect to the clonal group of the host strain, identified by chromosomal MLST, shows that each follows quite distinct patterns of dissemination in the K. pneumoniae population (Fig. 5).

\section{luc/iro lineages 1 and 2 are associated with two dominant K. pneumoniae virulence plasmids, KpVP-1 and KpVP-2}

Iuc/iro lineages 1 and 2 accounted for $64 \%$ of $K$. pneumoniae isolates carrying any aerobactin or salmochelin synthesis loci, and $88 \%$ of isolates carrying both. While it was not possible to resolve the complete sequences for all plasmids associated with these lineages, read mapping to pK2044 and Kp52.145 pII reference sequences strongly supported the presence of pK2044-like plasmids in iro1 $+i u c 1+$ genomes (mean plasmid coverage of $95.1 \%$, range 28.8-100\%; see Fig. 4) and Kp52.145 pII-like plasmids in iro2+iuc2+ genomes (mean plasmid coverage of $92.4 \%$, range $87.2-100 \%$; see Fig. 4). There were limited homologous regions shared between the two plasmids (Fig. 2), including the iro, iuc, rmpA and fec loci, and the $\operatorname{IncFIB}_{\mathrm{K}}$ replicon (Additional file 12). These shared regions were largely conserved across all isolates carrying iuc/iro lineages 1 or 2 ; the remaining regions unique to either pK2044 or Kp52.145 pII were largely conserved amongst

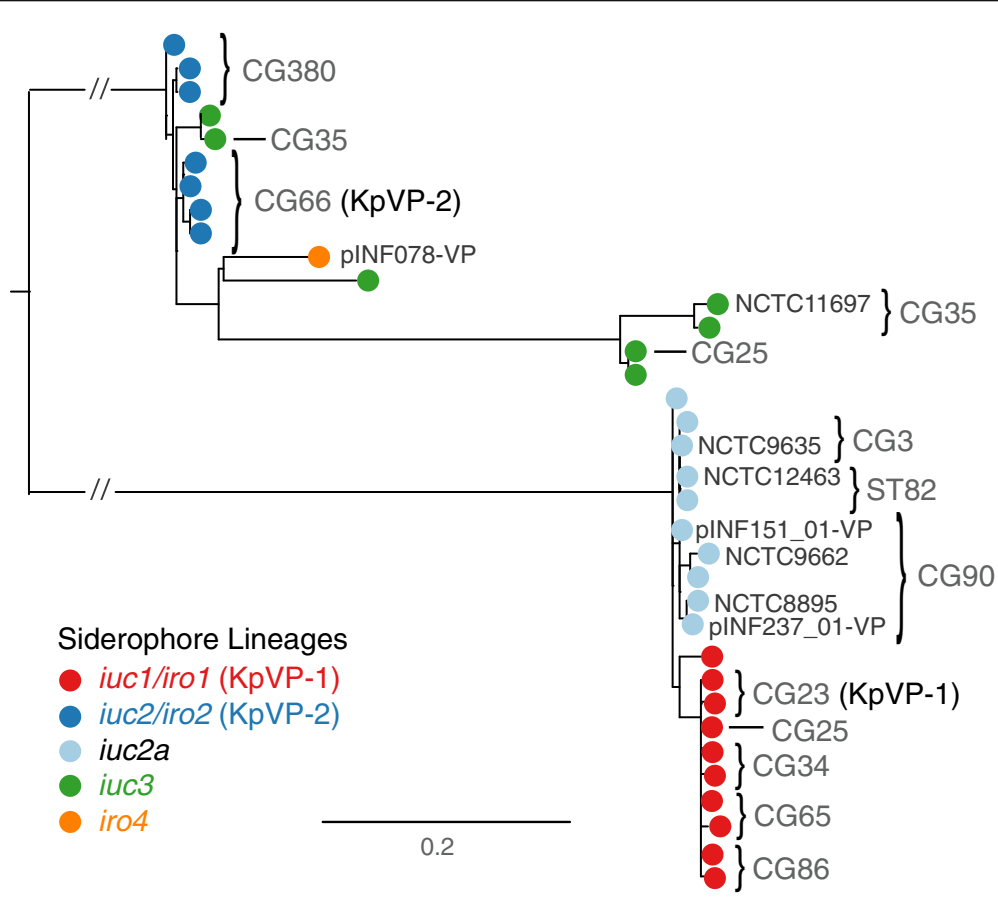

Fig. 3 Maximum likelihood phylogeny of representative $I_{C} F \mid B_{K}$ replicon sequences from isolates with iuc/iro plasmids. Each tip represents a unique $\mathrm{IncFIB}_{\mathrm{K}}$ replicon sequence (spanning repA, oriT, sopAB), coloured according to the iro/iuc lineage carried by the corresponding isolates as per inset legend. IncFIB $\mathrm{B}_{\mathrm{K}}$ sequences found in the representative plasmid sequences (shown in Fig. 2 and listed in Additional file 2) are labelled; tips/subclades are also annotated to indicate those found in common clonal groups (CG; see Fig. 5) 


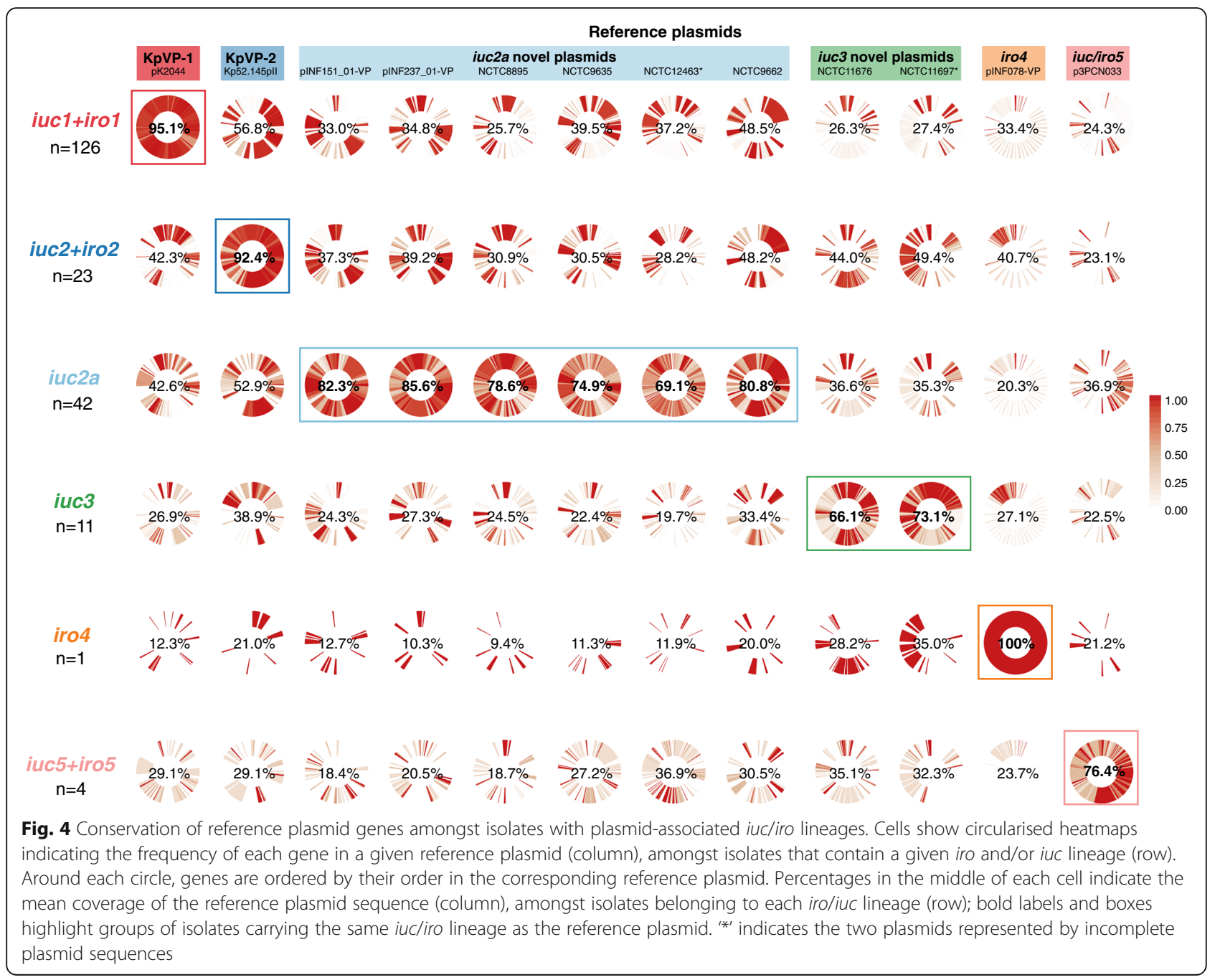

the isolates that carried lineage 1 or 2 loci, respectively (Fig. 4). Notably, the loci encoding heavy metal resistances against copper ( $p b r-p c o)$, silver (sil) and tellurite (ter XYW and terZABCDEF) were highly conserved amongst lineage 1 isolates but not present in any of the lineage 2 isolates (Additional file 12). As noted above, iuc/iro lineages 1 and 2 were also each associated with a distinct variant of the IncFIB $_{K}$ replicon sequence (Fig. 3). Hence, we define pK2044-like plasmids carrying iuc1 and iro1 loci as $K$. pneumoniae virulence plasmid type 1 (KpVP-1), with reference plasmid pK2044, and Kp52.145 pII-like plasmids carrying iuc2 and iro2 loci as $K$. pneumoniae virulence plasmid type 2 (KpVP-2). Both plasmid types typically carry at least one copy of $r m p A$; neither one carries genes associated with conjugation; hence, we assume they are not self-transmissible.

KpVP-1 and KpVP-2 showed distinct distributions within the K. pneumoniae population. KpVP-1 was present in $5.0 \%$ of all isolates and accounted for $74 \%$ of iuc+iro+ isolates. The KpVP-1 reference plasmid pK2044 originated from an ST23 isolate (CG23), and KpVP-1 was strongly associated with this and two other well-known hypervirulent clones CG65 and CG86, in which it was present at high prevalence (ranging from 79.0 to $96.4 \%$, see Fig. 5). KpVP-1 was also detected at low frequencies in other clones, including CG34, CG111, CG113 and CG25, suggesting it is mobile within the $K$. pneumoniae population (Fig. 5). KpVP-2 was present in $0.96 \%$ of all isolates and accounted for $14 \%$ of iuc+iro+ isolates. The KpVP-2 reference plasmid Kp52.145 pII originated from an ST66 isolate, and KpVP-2 was present in all isolates of the associated clonal group CG66 $(n=11)$ and also all isolates of CG380 ( $n=12)$ (Fig. 5).

An iuc lineage 2 variant (iuc2a) is associated with diverse plasmids with a KpVP-1-like IncFIB ${ }_{K}$ replicon

Iuc $2 a$ was identified in 43 isolates largely belonging to three clonal groups (ST3, $n=4$; CG90, $n=19$; ST82, $n=$ 19; ST382, $n=1$; see Fig. 5$)$, with the majority $(n=38$, 88.4\%) from the historical NCTC or Murray collections 


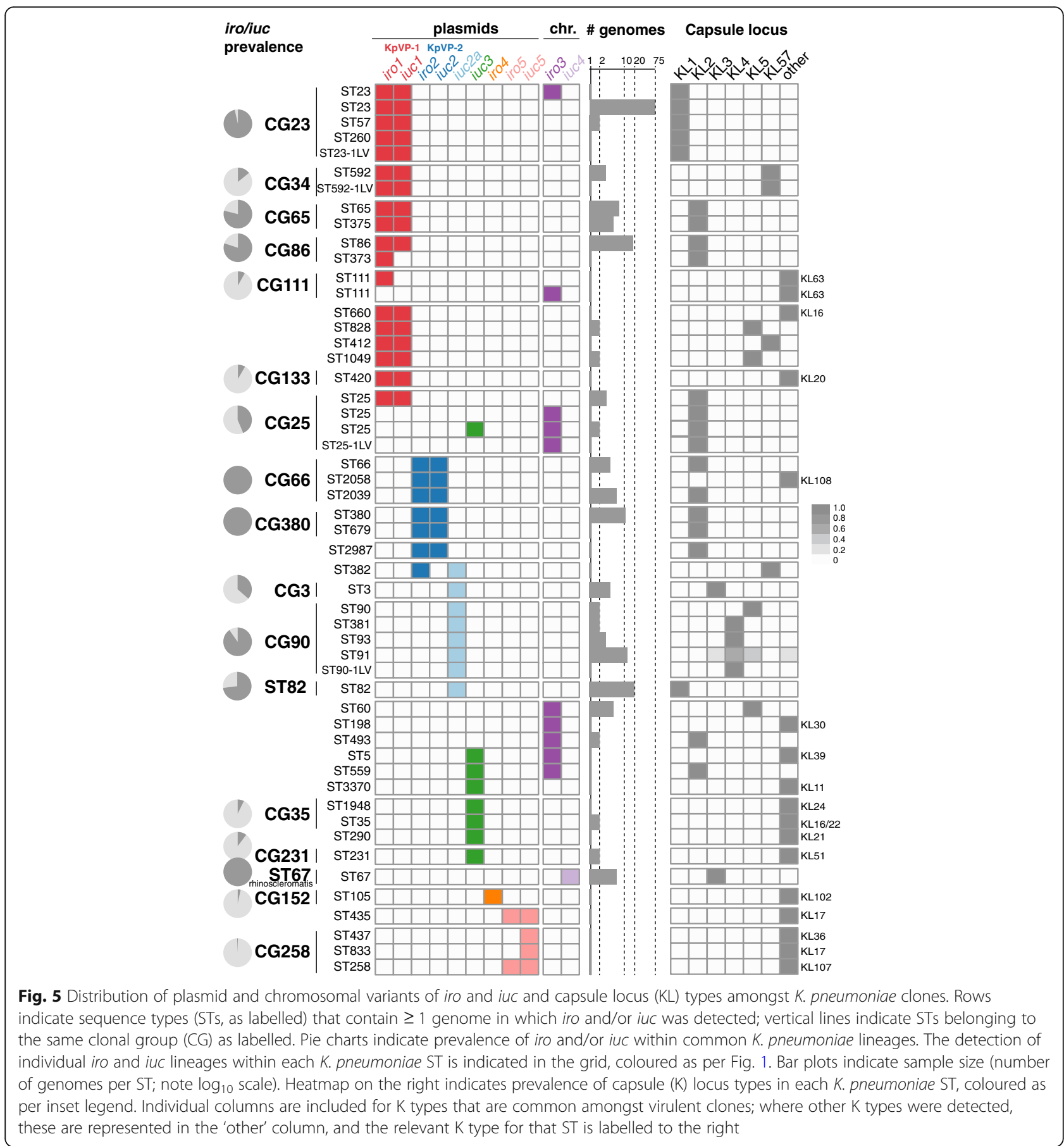

and isolated between 1932 and 1960 (Additional file 1). One of these isolates also carried iro 2 in addition to $i u c 2 a$, which in all other instances was only observed with iuc2 on KpVP-2. Provenance information was available for only 12 of the iuc $2 a+$ isolates (1 ST3, 9 CG90, 2 ST82); all of which originated from the human respiratory tract (3 nose, 1 throat, 7 sputum and 2 NCTC isolates recorded only as a respiratory tract). We used long-read sequencing to resolve plasmids in two novel $i u c 2 a+$ isolates from our own collection, INF151and INF237, which were both CG90 Australian hospital sputum isolates (summarised in Additional file 3). This yielded IncFIB $_{\mathrm{K}}$ plasmids in each genome, of size $138.1 \mathrm{kbp}$ and $133.7 \mathrm{kbp}$, respectively (accessions: pINF151_01-VP, QWFT01000004; pINF237_ 01-VP, CP032834). Both plasmids carried iuc2a and one $r m p A$ gene, but they differed slightly from one another in structure and gene content and differed substantially from the three complete $i u c 2 a+$ plasmid sequences available 
from NCTC isolates (ST3 and CG90; see Figs. 2 and 4). Only one of these plasmids (from NCTC 12463; incomplete) carried a conjugative transfer region (IncFII); hence, we predict most are not self-transmissible. Mapping of $i u c 2 a+$ genomes to each of the five representative iuc $2 a+$ plasmid sequences indicated a degree of conservation between plasmids in isolates belonging to the same $K$. pneumoniae clone, but none particularly well conserved across all iuc $2 a+$ isolates (Fig. 4, Additional file 13). However, all iuc $2 a+$ isolates formed a tight monophyletic cluster in the IncFIB $_{K}$ replicon tree (Fig. 3), consistent with recent shared plasmid ancestry followed by frequent structural and gene content changes. Notably, the iuc $2 a$-associated IncFIB $_{K}$ replicon sequences were closely related to those of KpVP-1 and distant from those of KpVP-2; hence, we hypothesise that iuc $2 a$ plasmids share an ancestor that was a mosaic including iuc2-related sequences from KpVP-2 and $\mathrm{IncFIB}_{\mathrm{K}}$ replicon sequences from KpVP-1.

\section{luc lineage 3 is mobilised by diverse plasmids carrying the IncFII $\mathrm{K}_{\mathrm{K}}$ conjugative transfer region}

Lineage iuc3 was detected in 11 isolates from diverse sources and chromosomal STs (Fig. 5) and was associated with three related variants of the $\operatorname{IncFIB}_{\mathrm{K}}$ replicon (Fig. 3). We identified one complete and one near-complete iuc3 plasmid sequences: a complete $189.8 \mathrm{~kb}$ plasmid from NCTC 11676 (isolated 1979, ST290) and a 155.4 kb contig from NCTC 11697 (isolated 1984, ST3370) (Fig. 2). The plasmids share around half of their gene content (96 kbp), including the $\mathrm{IncFII}_{\mathrm{K}}$ tra-trb conjugative transfer machinery, a fimbrial protein and the fec iron acquisition system in addition to iuc3 (Figs. 2 and 4, Additional file 2). Mapping to these sequences showed all iuc3+ isolates carried related plasmids with an IncFII $_{\mathrm{K}}$ tra-trb transfer region (Fig. 4, Additional file 12).

\section{Complete sequence of an iro 4 plasmid}

Lineage iro4 was identified in a single hospital UTI isolate INF078 (ST105) from Australia, whose genome sequence we completed using long reads (replicons summarised in Additional file 3). Hybrid assembly using short and long reads resolved a 399,913 kbp plasmid, pINF078-VP (accession CP032832) which carried multiple copies of iro4, the $\mathrm{IncFIB}_{\mathrm{K}}$ replicon (similar to the KpVP-2 variant, see Fig. 5) and the IncFII $_{\mathrm{K}}$ replicon and tra-trb transfer region (Fig. 2). As noted above, the iro4 locus is more closely related to Enterobacter iro than to other K. pneumoniae iro in terms of both structure (including the $i r o E$ gene; see Fig. 1b, Additional file 14) and sequence (Additional file 10), suggesting it has been transferred from Enterobacter into a $K$. pneumoniae IncFIB $_{\mathrm{K}} / \mathrm{FII}_{\mathrm{K}}$ plasmid backbone. pINF078-VP harboured multiple tandem copies of a 17,129 bp region containing
iroBCDEN and 12 other genes of unknown function (Additional file 14). Long-read sequences (up to $70 \mathrm{kbp}$ ) spanning the non-repeat and repeat region of pINF078-VP confirmed at least $n=3$ copies of the $17 \mathrm{kbp}$ repeated sequence, whose mean read depth in the Illumina sequence data was 13.3 times that of the rest of the plasmid sequence, suggesting approximately 13 tandem copies.

\section{luc/iro lineage 5 loci are associated with plasmids originating from $E$. coli}

Four $K$. pneumoniae isolates carried the E. coli variant iuc5; two of these also carried the E. coli variant iro5 (see species trees in Additional file 10). Three iuc5+ isolates (including one with iro5) belonged to the globally disseminated, carbapenemase-producing $K$. pneumoniae CG258 (ST258, KPC+; ST437, KPC+; ST833, KPC-) and carried several AMR genes. Unfortunately, all four iuc5+ genomes were sourced from public databases and were available in draft form only, and the complete plasmid sequences could not be resolved. However, the iuc5+ contig sequences from $K$. pneumoniae share close homology with iuc5+iro5+ IncFII conjugative plasmids from $E$. coli that also carry AMR genes (e.g. p3PCN033, CP006635.1; D3 plasmid A, CP010141.1). Notably, all iuc5 contigs from K. pneumoniae shared $>75 \%$ coverage and $98.19-99.95 \%$ identity to the p3PCNO33 reference plasmid.

\section{Discussion}

This study reveals significant genetic diversity underlying the biosynthesis of aerobactin and salmochelin in $K$. pneumoniae but shows the distribution of iuc and iro locus variants is highly structured within the population. Our data indicate that most of the burden of these hypervirulence-associated siderophores in the K. pneumoniae population is associated with two dominant virulence plasmids, which we define here as KpVP-1 and KpVP-2, that differ in terms of gene content (Fig. 2) and are each associated with co-segregating sequences of the non-self-transmissible IncFIB $_{\mathrm{K}}$ replicon, iuc and iro loci (Figs. 1 and 3). These dominant virulence plasmid types are each represented by one of the previously characterised $K$. pneumoniae virulence plasmids $[18,20]$, pK2044 (KpVP-1, encoding iro1 and iuc1) and Kp152.145pII (KpVP-2, encoding iro2 and iuc2); both also carry hypermucoidy determinants, and together, they account for $74 \%$ and $14 \%$ of the iuctiro+ K. pneumoniae genomes analysed. Importantly, our data indicate that each of these common virulence plasmid variants is maintained at high prevalence in a small number of known hypervirulent clones: KpVP-1 in CG23 (96\%, including pK2044 [18]), CG86 (80\%, including pLVPK [19]) and CG65 (79\%); KpVP-2 in CG66 (100\%, including Kp152.145pII) and CG380 (100\%) (Fig. 5). This suggests that both plasmid types can persist for long 
periods within a host bacterial lineage as it undergoes clonal expansion; indeed, our recent study of the evolutionary history of CG23 indicates that KpVP-1 has been maintained in this clonally expanding lineage for at least a century [22]. The lack of conjugation machinery is likely an important variable contributing to clonal expansion being the primary mode of dispersal over horizontal gene trasfer, although notably, we also detected KpVP-1 at low prevalence in numerous other $K$. pneumoniae lineages and KpVP-2 at low prevalence in one other lineage, suggesting the possibility of wider dissemination of both plasmid types by occasional transfer to new lineages (Fig. 5). Given the stability of the plasmids observed in several clonal groups, we speculate that some of these transfer events will result in the emergence of novel hypervirulent strains that can stably maintain the plasmid into the future. In contrast, the non-plasmid form of iro (iro3, occasionally integrated into the chromosomes of $K$. pneumoniae via ICEKp1) was found at low prevalence $(<0.5 \%)$ and included just 1 of the 79 ST23 isolates analysed (NTUH-K2044, in which ICEKp1 was first described), 1/1 ST5, 1/21 ST111 (13\%), 1/2 ST198, 2/15 CG25, 2/2 ST493 and 5/5 ST60. Hence, while ICEKp1 is somewhat dispersed in the K. pneumoniae population, it shows little evidence of stability within lineages, consistent with our previous observations regarding ICE $K p$ in general [8].

We also detected several novel iuc+ or iro+ plasmid types, the most common being the group of iuc $2 a$ plasmids (21\% of all iuc+ isolates) that were detected in respiratory isolates from CG3, CG82 and CG90 and mostly originated from historical collections [51]. Interestingly, these combine an iuc sequence closely related to that of KpVP-2 (Fig. 1) with an IncFIB $_{\mathrm{K}}$ replicon sequence very close to that of KpVP-1 (Fig. 3) and showed substantial mosaicism and gene content variation (Figs. 2 and 4). The iuc3 lineage was also quite common amongst the novel plasmid types (5.3\% of all iuc+ isolates) and associated with a variety of diverse plasmids, most of which carried the IncFII tra-trb conjugative transfer region and thus are likely self-transmissible (Figs. 2 and 4). It is notable that $i u c 2 a$ and $i u c 3$ plasmids were not only relatively rare in the bacterial population but also showed less evidence of stable maintenance within $K$. pneumoniae lineages (Fig. 5) and lower stability of gene content (Fig. 2) than the dominant KpVP-1 and KpVP-2 plasmids (Fig. 4). The position of $i u c 2 a$ and $i u c 3$ in the iuc trees (Fig. 1, Additional file 10) suggests that both are derive from other $K$. pneumoniae loci; hence, we speculate it is the properties of the plasmids mobilising these loci, and not the siderophore biosynthesis loci themselves, that makes these variants less widespread in the K. pneumoniae population. This variation in gene content may be a consequence of self-transmissibility, exposing the plasmids to a wider gene pool of host bacteria and providing opportunities for gene content diversification, which could potentially include AMR genes. Notably, the iuc3 plasmids carry an arsenal of additional virulence loci involved in iron metabolism and resistance to heavy metals, reminiscent of KpVP-1 (Fig. 2).

The other novel plasmids appear to derive from outside $K$. pneumoniae (Fig. 1, Additional file 10). Most concerning are the four $E$. coli-derived plasmids we detected carrying iuc5 (and occasionally iro5) in the USA and Brazil, three of which were found in the MDR hospital outbreak-associated clone CG258. Whether these aerobactin plasmids harbour AMR genes as they do in $E$. coli is not currently resolvable; however, it seems that conjugative E. coli plasmids such as D3 plasmid A do have the potential to deliver hypervirulence and multidrug resistance to K. pneumoniae strains in a single step. A recent study of $K$. pneumoniae submitted to Public Health England used PCR to screen for isolates carrying both carbapenemase genes and $\operatorname{rmp} A$, as a marker of the virulence plasmid, and identified a plasmid harbouring iuc, rmpA, rmpA2 and the AMR genes sul1, sul2, armA, $d f r A 5, m p h(A)$ and $a p h\left(3^{\prime}\right)-V I b$ [28]. To our knowledge, this is the first report of a complete sequence of a $K$. pneumoniae plasmid harbouring both AMR and virulence genes. The isolate (ST147) was not included in our original screen; however, subsequent analysis using Kleborate plus manual inspection of the plasmid sequence reveals it carries iuc1 (AbST63, a novel single locus variant of AbST1 which is typical of hypervirulent clones CG23, CG65 and CG86) and appears to be a mosaic carrying sequences from KpVP-1 (40\% coverage), an IncFII tra-trb conjugative transfer region and transposons carrying AMR genes.

The presence of aerobactin synthesis loci in the iuc5+ K. pneumoniae isolates we identified here was not reported in the original studies $[52,53]$, and thus, it is not known whether they actually produce aerobactin or show enhanced virulence. This highlights the need to raise awareness of the iuc and iro loci as potentially clinically relevant hypervirulence factors and to screen for them in isolates and genome data. The latter, we aim to facilitate via the genotyping schemes established here, which can be used to easily screen new genome assemblies using Kleborate (https://github.com/katholt/Klebo rate/) or BIGSdb-Kp (http://bigsdb.pasteur.fr/klebsiella/ klebsiella.html), or new short-read data sets using SRST2 (https://github.com/katholt/srst2). PCR primers suitable for screening for iro and iuc can be found in Lee et al. [54]. Notably, many studies rely on the hypermucoidy phenotype to identify hypervirulent strains; however, this is dependent on growth conditions [55], and recent studies indicate that aerobactin synthesis is a more important virulence determinant $[13,14,16]$. Our data suggest that hypermucoidy screening would typically pick 
up most of the common aerobactin plasmids KpVP-1, KpVP-2 and iuc $2 a+$ plasmids, but not those carrying $i u c 3$ or the $i u c 5$ plasmids from E. coli. Additionally, it is important not to conflate the presence of the core chromosomal receptor gene iutA with the ability to synthesise aerobactin, which is encoded in the iuc locus [6]. False-positive detection of the aerobactin locus version of iutA can be avoided by using an identity threshold of $<20 \%$ divergence. Tellurite resistance has also been suggested as a phenotypic screen to identify hypervirulent isolates of CG23, CG65 and CG86 [56]; our data confirm this is a good marker for KpVP-1 (92.6\% carry ter) but not for other aerobactin plasmid types (Additional file 12).

\section{Conclusions}

Our results illuminate that distinct virulence plasmid variants are associated with the various hypervirulent $K$. pneumoniae lineages but also highlight that these alongside other plasmids and MGEs can shuttle aerobactin and salmochelin synthesis loci to other lineages, threatening the emergence of novel hypervirulent strains. Indeed, reports of MDR clones acquiring iuc plasmids appear to be increasing in incidence, particularly in China [27, 29, 57-59] and have been associated with increased morbidity and mortality. The AbST and SmST typing schemes developed in this study provide an important resource to identify and monitor the movement of iro and iuc loci and associated MGEs in K. pneumoniae genomes; which will be important to detect and contain these emerging threats. Genotyping with our tools reveals the iuc plasmid identified in the recently reported fatal hospital outbreak of carbapenemase-producing ST11 in Beijing is a variant of KpVP-1 that carries iuc1 (AbST1) and a single copy of rmpA but lacks the iro locus [27]. In this strain, the aerobactin plasmid does not carry any AMR determinants; the carbapenemase gene $b l a_{\mathrm{KPC}}$ and several other AMR genes were located on other plasmids. Concerningly, the ability for the virulence plasmids to be maintained in $K$. pneumoniae lineages suggests that once established in the MDR hospital outbreak-associated clones, they may become quite stable. The initial report of $i u c+$ KPC+ ST11 in China prompted multiple other groups to report the detection of the same strain in their hospitals [60-62], suggesting this strain may indeed be emerging as a persistently hypervirulent and MDR form of K. pneumoniae. Genomic surveillance and control of the spread of such 'dual-risk' strains, or indeed even plasmids combining both characteristics of MDR and hypervirulence, clearly needs to be reinforced; the present work will bolster efforts to understand and limit the emergence of infections caused by K. pneumoniae strains carrying the high virulence determinants aerobactin and salmochelin.

\section{Additional files}

Additional file 1: Strain information for genomes included in this study. (XLS $426 \mathrm{~kb}$ )

Additional file 2: General features of reference plasmids or incomplete plasmid sequences carrying iro and/or iuc. (DOC $48 \mathrm{~kb}$ )

Additional file 3: Summary of replicon sequences from isolates INF151, INF237 and INF078. (DOC 39 kb)

Additional file 4: Aerobactin sequence types (AbSTs) and corresponding alleles. (TXT $2 \mathrm{~kb}$ )

Additional file 5: Salmochelin sequence types (SmSTs) and corresponding alleles. (TXT $1 \mathrm{~kb}$ )

Additional file 6: Representative Enterobacterales genome sequences included in iro and iuc phylogenetic analysis. (CSV 6 kb)

Additional file 7: Phylogenetic relationships between the predicted amino acid sequences encoded by aerobactin (iuc) and salmochelin (iro) locus sequence types. Each tip represents a translated amino acid sequence for an aerobactin sequence type (AbST, in a) or salmochelin sequence type (SmST, in b). Lineages defined from nucleotide sequences (see tree in Fig. 1) are highlighted and labelled. (PDF 135 kb)

Additional file 8: Single nucleotide variants and nucleotide divergence (\%) observed within (shaded in grey) and between the aerobactin-encoding iuc lineages. (DOC $33 \mathrm{~kb}$ )

Additional file 9: Single nucleotide variants and nucleotide divergence (\%) observed within (shaded in grey) and between the salmochelin-encoding iro lineages. (DOC $30 \mathrm{~kb}$ )

Additional file 10: Phylogenetic trees for salmochelin and aerobactin encoding iuc locus in K. pneumoniae and other Enterobacterales bacteria. Trees represent show a midpoint-rooted maximum likelihood phylogeny for representative sequences identified in various Enterobacterales species (listed in Additional file 6). Tip colours indicate the genetic context of the locus: black = plasmid, red = chromosome. K. pneumoniae iro lineages defined in Fig. 1 are coloured; other species-specific clades are highlighted in grey; individually labelled tips within highlighted clades indicate exceptions to the species label of the clade. Salmochelin trees were inferred using the iro $B$ gene alone (panel a), which show a highly divergent form in Salmonella. Panel (b) shows a tree inferred from all four genes of the typical K. pneumoniae iro locus (iroBCDN), excluding the distantly related Salmonella variant, to increase resolution within the group containing Klebsiella. Similarly, aerobactin trees were inferred using the iucB gene alone (panel c) to show the overall structure, and separately for the full set of genes in the K. pneumoniae locus (iucABCD, iutA) to provide greater resolution within the group containing Klebsiella (panel d). (PDF $297 \mathrm{~kb}$ )

Additional file 11: Summary of aerobactin-encoding iuc and salmochelin-encoding iro loci BLAST hit. (CSV $1 \mathrm{~kb}$ )

Additional file 12: Prevalence of virulence loci and plasmid replication loci amongst isolates with virulence plasmids. (CSV $1 \mathrm{~kb}$ )

Additional file 13: Conservation of coding sequences from KpVP-2 and iuc2a+ reference plasmids amongst isolates carrying plasmid-encoded iuc2 or iuc2a loci. Cells show circularised heatmaps indicating the frequency of each gene in a given reference plasmid (column), amongst isolates of a given chromosomal sequence type (ST) or clonal group (CG) (rows) that carry either iuc2 (CG66, CG380) or iuc2a (others). Around each circle, genes are ordered by their order in the corresponding reference plasmid. (PDF $2864 \mathrm{~kb}$ )

Additional file 14: Genetic structure of $17 \mathrm{kbp}$ repeat region in plasmid pINF078-VP and the chromosomally-encoded E. cloacae iro region. Shaded area indicates a homologous region of $95 \%$ nucleotide identity shared between the two sequences. Coding sequences are represented by the arrows and coloured according to the closest Enterobacteriaceae species match as indicated in the legend. (PDF $172 \mathrm{~kb}$ )

\section{Abbreviations}

AbST: Aerobactin sequence type; AMR: Antimicrobial resistance; CA: Community-associated; CG: Clonal group; Cl: Confidence interval; Ent: Enterobactin; HA: Healthcare-associated; HGT: Horizontal gene transfer; 
ICEs: Integrative and conjugative elements; Iro: Salmochelin; luc: Aerobactin; MDR: Multidrug-resistant; MGEs: Mobile genetic elements; MLST: Multi-locus sequence typing; OR: Odds ratio; SmST: Salmochelin sequence type; ST: Sequence type; Ybt: Yersiniabactin

\section{Acknowledgements}

We thank the team of the curators of the Institut Pasteur MLST system (Paris, France) for importing novel alleles, profiles and/or isolates at http///bigsdb.pasteur.fr.

\section{Funding}

This work was funded by the National Health and Medical Research Council (NHMRC) of Australia (project \#1043822), a Senior Medical Research Fellowship from the Viertel Foundation of Australia and the Bill and Melinda Gates Foundation of Seattle, USA.

\section{Availability of data and materials}

All whole-genome sequences analysed in this study are publicly available in NCBI or the NCTC 3000 Project website (https://www.sanger.ac.uk/resources/ downloads/bacteria/nctc/), accession numbers are listed in Additional file 1. Complete genome sequences generated for this study (summarised in Additional file 3) have been deposited in NCBI GenBank under accessions OWFT01000001-QWFT01000009, and CP032831-CP032838. Accession numbers for the 12 reference plasmid sequences are listed in Additional file 2; the set of annotated sequences and the Mauve multiple alignment of these sequences are also deposited in FigShare (doi: https://doi.org/10.6084/m9.figshare.6839981). The aerobactin and salmochelin MLST schemes are available in the K. pneumoniae BIGSdb database (http://bigsdb.pasteur.fr/klebsiella/ klebsiella.html) and in the Kleborate distribution (https://github.com/katholt/ Kleborate)

\section{Authors' contributions}

MMCL performed the majority of data analyses and wrote the paper together with $\mathrm{KEH}$. RRW, KLW, SB and KEH contributed additional data analysis, visualisation and interpretation. SB incorporated the novel MLST schemes into the BIGSdb. RRW and KEH wrote the code. AJ contributed clinical isolates, data and interpretations. LMJ performed the DNA extraction and nanopore sequencing. All authors edited and approved the final paper.

\section{Ethics approval and consent to participate}

Not applicable.

\section{Consent for publication}

Not applicable.

\section{Competing interests}

The authors declare that they have no competing interests.

\section{Publisher's Note}

Springer Nature remains neutral with regard to jurisdictional claims in published maps and institutional affiliations.

\section{Author details}

'Department of Biochemistry and Molecular Biology, Bio21 Molecular Science and Biotechnology Institute, University of Melbourne, Parkville, Victoria 3010, Australia. ${ }^{2}$ Biodiversity and Epidemiology of Bacterial Pathogens, Institut Pasteur, 75015 Paris, France. ${ }^{3}$ Department of Infectious Diseases and Microbiology Unit, The Alfred Hospital, Melbourne, Victoria 3004, Australia. ${ }^{4}$ London School of Hygiene \& Tropical Medicine, London WC1E 7HT, UK.

Received: 24 July 2018 Accepted: 12 October 2018

Published online: 29 October 2018

\section{References}

1. Podschun R, Ullmann U. Klebsiella spp. as nosocomial pathogens: epidemiology, taxonomy, typing methods, and pathogenicity factors. Clin Microbiol Rev. 1998;11(4):589-603.

2. Martin RM, Bachman MA. Colonization, infection, and the accessory genome of Klebsiella pneumoniae. Front Cell Infect Microbiol. 2018;8(4). https://doi.org/10.3389/fcimb.2018.00004.

3. Holt KE, Wertheim H, Zadoks RN, Baker S, Whitehouse CA, Dance D, et al. Genomic analysis of diversity, population structure, virulence, and antimicrobial resistance in Klebsiella pneumoniae, an urgent threat to public health. Proc Natl Acad Sci U S A. 2015:112(27):E3574-81.

4. Ramirez MS, Traglia GM, Lin DL, Tran T, Tolmasky ME. Plasmid-mediated antibiotic resistance and virulence in gram-negatives: the Klebsiella pneumoniae paradigm. Microbiol Spectr. 2014;2(5):1-15.

5. Gorrie CL, Mirceta M, Wick RR, Edwards DJ, Strugnell RA, Pratt N, et al. Gastrointestinal carriage is a major reservoir of K. pneumoniae infection in intensive care patients. Clin Infect Dis. 2017;65(2):208-15.

6. Runcharoen C, Moradigaravand D, Blane B, Paksanont S, Thammachote J, Anun $\mathrm{S}$, et al. Whole genome sequencing reveals high-resolution epidemiological links between clinical and environmental Klebsiella pneumoniae. Genome Med. 2017;9(1):6. https://doi.org/10.1186/s13073-0170397-1.

7. Lin TL, Lee CZ, Hsieh PF, Tsai SF, Wang JT. Characterization of integrative and conjugative element ICEKp 1-associated genomic heterogeneity in a Klebsiella pneumoniae strain isolated from a primary liver abscess. J Bacteriol. 2008:190(2):515-26.

8. Lam MMC, Wick RR, Wyres KL, Gorrie C, Judd LM, Jenney A, et al. Genetic diversity, mobilisation and spread of the yersiniabactin-encoding mobile element ICEKp in Klebsiella pneumoniae populations. Microb Genom. 2018; 4(9). https://doi.org/10.1099/mgen.0.000196.

9. Nassif X, Sansonetti PJ. Correlation of the virulence of Klebsiella pneumoniae $\mathrm{Kl}$ and $\mathrm{K} 2$ with the presence of a plasmid encoding aerobactin. Infect Immun. 1986;54(3):603-8.

10. Müller S, Valdebenito M, Hantke K. Salmochelin, the long-overlooked catecholate siderophore of Salmonella. Biometals. 2009;22(4):691-5.

11. Goetz DH, Holmes MA, Borregaard N, Bluhm ME, Raymond KN, Strong RK. The neutrophil lipocalin NGAL is a bacteriostatic agent that interferes with siderophore-mediated iron acquisition. Mol Cell. 2002;10(5):1033-43.

12. Fischbach MA, Lin $H$, Zhou L, Yu Y, Abergel RJ, Liu DR, et al. The pathogenassociated iroA gene cluster mediates bacterial evasion of lipocalin 2. Proc Natl Acad Sci U S A. 2006;103(44):16502-7.

13. Russo TA, Olson R, Macdonald U, Beanan J, Davidson BA. Aerobactin, but not yersiniabactin, salmochelin, or enterobactin, enables the growth/survival of hypervirulent (hypermucoviscous) Klebsiella pneumoniae ex vivo and in vivo. Infect Immun. 2015;83(8):3325-33.

14. Russo TA, Olson R, Macdonald U, Metzger D, Maltese LM, Drake EJ. Aerobactin mediates virulence and accounts for increased siderophore production under iron-limiting conditions by hypervirulent (hypermucoviscous) Klebsiella pneumoniae. Infect Immun. 2014;82(6):2356-67.

15. Konopka K, Bindereif A, Neilands J. Aerobactin-mediated utilization of transferrin iron. Biochemistry. 1982;21(25):6503-8.

16. Russo TA, Olson R, Fang C-T, Stoesser N, Miller M, Hutson A, et al. Identification of biomarkers for the differentiation of hypervirulent Klebsiella pneumoniae from classical K. pneumoniae. J Clin Microbiol. 2018. https://doi.org/10.1128/ JCM.00776-18.

17. Putze J, Hennequin C, Nougayrède JP, Zhang W, Homburg S, Karch H, et al. Genetic structure and distribution of the colibactin genomic island among members of the family Enterobacteriaceae. Infect Immun. 2009;77(11):4696-703.

18. Wu KM, Li NH, Yan J, Tsao N, Liao TL, Tsai HC, et al. Genome sequencing and comparative analysis of Klebsiella pneumoniae NTUHK2044, a strain causing liver abscess and meningitis. J Bacteriol. 2009; 191(14):4492-501.

19. Chen $Y$, Chang $H$, Lai $Y$, Pan C, Tsai S. Sequencing and analysis of the large virulence plasmid pLVPK of Klebsiella pneumoniae CG43. Gene. 2004;337: 189-98.

20. Lery LM, Frangeul L, Tomas A, Passet V, Almeida AS, Bialek-Davenet S, et al. Comparative analysis of Klebsiella pneumoniae genomes identifies a phospholipase D family protein as a novel virulence factor. BMC Biol. 2014; 12(1):41.

21. Nassif X, Fournier J, Arondel J, Sansonetti PJ. Mucoid phenotype of Klebsiella pneumoniae is a plasmid-encoded virulence factor. Infect Immun. 1989; 57(2):546-52.

22. Lam MM, Wyres KL, Duchêne S, Wick RR, Judd LM, Gan Y, et al. Population genomics of hypervirulent Klebsiella pneumoniae clonal-group 23 reveals early emergence and rapid global dissemination. Nat Comms. 2018. https:// doi.org/10.1101/225359.

23. Struve C, Roe CC, Stegger M, Stahlhut SG, Hansen DS, Engelthaler DM, et al. Mapping the evolution of hypervirulent Klebsiella pneumoniae. MBio. 2015;6(4):1-12. 
24. Peng H, Wang P, Wu J, Chiu C, Chang H. Molecular epidemiology of Klebsiella pneumoniae. Zhonghua Min Guo Wei Sheng Wu Ji Mian Yi Xue Za Zhi. 1991;24:264-71.

25. Bialek-davenet S, Criscuolo A, Ailloud F, Passet V, Jones L, Garin B, et al. Genomic definition of hypervirulent and multidrug-resistant Klebsiella pneumoniae clonal groups. Emerg Infect Dis. 2014;20(11):1812-20.

26. Shon AS, Russo TA. Hypervirulent Klebsiella pneumoniae: the next superbug? Future Microbiol. 2012;7(6):669-71.

27. Gu D, Dong N, Zheng Z, Lin D, Huang M, Wang L, et al. A fatal outbreak of ST11 carbapenem-resistant hypervirulent Klebsiella pneumoniae in a Chinese hospital: a molecular epidemiological study. Lancet Infect Dis. 2018. https:// doi.org/10.1016/S1473-3099(17)30489-9.

28. Turton JF, Payne Z, Coward A, Hopkins K, Turton J, Doumith M, et al. Virulence genes in isolates of Klebsiella pneumoniae from the UK during 2016, including among carbapenemase gene-positive hypervirulent K1-ST23 and "non-hypervirulent" types ST147, ST15 and ST383. J Med Microbiol. 2018;67(1):118-28

29. Chen L, Kreiswirth BN. Convergence of carbapenem-resistance and hypervirulence in Klebsiella pneumoniae. Lancet Infect Dis. 2018. https://doi. org/10.1016/S1473-3099(17)30517-0

30. Cheong HS, Chung DR, Lee C, Kim SH, Kang C, Peck KR. Emergence of serotype K1 Klebsiella pneumoniae ST23 strains co-producing the DHA-1 and an extended-spectrum beta-lactamase in Korea. Antimicrob Resist Infect Control. 2016. https://doi.org/10.1186/s13756-016-0151-2.

31. Shin J, Ko KS. Single origin of three plasmids bearing bla CTX-M-15 from different Klebsiella pneumoniae clones. J Antimicrob Chemother. 2014;69: 969-72.

32. Wyres KL, Wick RR, Gorrie C, Jenney A, Follador R, Thomson NR, et al. Identification of Klebsiella capsule synthesis loci from whole genome data. Microb Genom. 2016. https://doi.org/10.1099/mgen.0.000102.

33. Wick RR, Judd LM, Gorrie C, Holt KE. Unicycler: resolving bacterial genome assemblies from short and long sequencing reads. PLoS Comput Biol. 2017: 13(6):e1005595.

34. Bankevich A, Nurk S, Antipov D, Gurevich AA, Dvorkin M, Kulikov AS, et al SPAdes: a new genome assembly algorithm and its applications to singlecell sequencing. J Comp Biol. 2012;19(5):455-77.

35. Seemann T. Prokka: rapid prokaryotic genome annotation. Bioinformatics. 2014;30(14):2068-9.

36. Wick RR, Judd LM, Gorrie CL, Holt KE. Completing bacterial genome assemblies with multiplex MinION sequencing. Microb Genom. 2017:3:1-7.

37. Diancourt L, Passet V, Verhoef J, Grimont PAD, Brisse S. Multilocus sequence typing of Klebsiella pneumoniae nosocomial isolates. J Clin Microbiol. 2005; 43(8):4178-82

38. Inouye M, Dashnow H, Raven L-A, Schultz MB, Pope BJ, Tomita T, et al. SRST2: rapid genomic surveillance for public health and hospital microbiology labs. Genome Med. 2014;6(11):90.

39. Wick RR, Heinz E, Holt KE, Wyres KL. Kaptive web: user-friendly capsule and lipopolysaccharide serotype prediction for Klebsiella genomes. J Clin Microbiol. 2018. https://doi.org/10.1128/JCM.00197-18.

40. Carattoli A, Zankari E, García-fernández A, Larsen V, Lund O, Villa L, et al. In silico detection and typing of plasmids using PlasmidFinder and plasmid multilocus sequence typing. Antimicrob Agents Chemother. 2014;58(7): 3895-903.

41. Wick RR, Schultz MB, Zobel J, Holt KE. Bandage: interactive visualization of de novo genome assemblies. Bioinformatics. 2015;31:3350-2.

42. Edgar RC. MUSCLE: multiple sequence alignment with high accuracy and high throughput. Nucleic Acids Res. 2004;32(5):1792-7.

43. Stamatakis A. RAxML-VI-HPC: maximum likelihood-based phylogenetic analyses with thousands of taxa and mixed models. Bioinformatics. 2006; 22(21):2688-90.

44. Carattoli A. Resistance plasmid families in Enterobacteriaceae. Antimicrob Agents Chemother. 2009;53(6):2227-38.

45. Yi H, Xi Y, Liu J, Wang J, Wu J, Xu T, et al. Sequence analysis of pKF3-70 in Klebsiella pneumoniae: probable origin from R100-like plasmid of Escherichia coli. PLoS One. 2010;5(1):e8601.

46. Darling ACE, Mau B, Blattner FR, Perna NT. Mauve: multiple alignment of conserved genomic sequence with rearrangements. Methods. 2004;14: 1394-403.

47. Guy L, Kultima J, Andersson S. genoPlotR: comparative gene and genome visualization in R. Bioinformatics. 2010;26(18):2334-5.
48. Langmead B, Saizberg SL. Fast gapped-read alignment with Bowtie 2. Nat Methods. 2012:9:357-9.

49. Liu C, Zheng H, Yang M, Xu Z, Wang X, Wei L, et al. Genome analysis and in vivo virulence of porcine extraintestinal pathogenic Escherichia coli strain PCN033. BMC Genomics. 2015. https://doi.org/10.1186/s12864-015-1890-9.

50. Capone A, Giannella M, Fortini D, Giordano A, Meledandri M, Ballardini M, et al. High rate of colistin resistance among patients with carbapenemresistant Klebsiella pneumoniae infection accounts for an excess of mortality. Clin Microbiol Infect. 2012. https://doi.org/10.1111/1469-0691.12070.

51. Wand ME, Baker KS, Benthall G, McGregor H, McCowen JWI, Deheer-Graham A, et al. Characterization of pre-antibiotic era Klebsiella pneumoniae isolates with respect to antibiotic/disinfectant susceptibility and virulence in Galleria mellonella. Antimicrob Agents Chemother. 2015;59(7):3966-72.

52. Bowers JR, Kitchel B, Driebe EM, MacCannell DR, Roe C, Lemmer D, et al. Genomic analysis of the emergence and rapid global dissemination of the clonal group 258 Klebsiella pneumoniae pandemic. PLoS One. 2015;10(7):1-24.

53. Davis GS, Waits K, Nordstrom L, Weaver B, Aziz M, Gauld L, et al. Intermingled Klebsiella pneumoniae populations between retail meats and human urinary tract infections. Clin Infect Dis. 2015;61:892-9.

54. Lee IR, Molton JS, Wyres KL, Gorrie C, Wong J, Hoh CH, et al. Differential host susceptibility and bacterial virulence factors driving Klebsiella liver abscess in an ethnically diverse population. Sci Rep. 2016. https://doi.org/10. 1038/srep29316.

55. Catalán-Nájera JC, Garza-Ramos U, Barrios-Camacho H. Hypervirulence and hypermucoviscosity: two different but complementary Klebsiella spp. phenotypes? Virulence. 2017; doi: https://doi.org/10.1080/21505594.2017.1317412

56. Passet $\mathrm{V}$, Brisse $\mathrm{S}$. Association of tellurite resistance with hypervirulent clonal groups of Klebsiella pneumoniae. J Clin Microbiol. 2015;53(4):1380-2.

57. Zhan L, Wang S, Guo Y, Jin Y, Duan J, Hao Z, et al. Outbreak by hypermucoviscous Klebsiella pneumoniae ST11 isolates with carbapenem resistance in a tertiary Hospital in China. Front Cell Infect Microbiol. 2017. https://doi.org/10.3389/fcimb.2017.00182.

58. Zhang Y, Zeng J, Liu W, Zhao F, Hu Z, Zhao C, et al. Emergence of a hypervirulent carbapenem-resistant Klebsiella pneumoniae isolate from clinical infections in China. J Inf Secur. 2015. https://doi.org/10.1016/j.jinf 2015.07.010

59. Araújo BF, Ferreira ML, De Campos PA, Royer S, Gonçalves IR, Fernandes MR, et al. Hypervirulence and biofilm production in KPC-2-producing Klebsiella pneumoniae CG258 isolated in Brazil. J Med Microbiol. 2018;67:523-8.

60. Du P, Zhang Y, Chen C. Emergence of carbapenem-resistant hypervirulent Klebsiella pneumoniae. Lancet Infect Dis. 2018. https://doi.org/10.1016/S14733099(17)30625-4.

61. Yao H, Qin S, Chen S, Shen J, Du X-D. Emergence of carbapenem-resistant hypervirulent Klebsiella pneumoniae. Lancet Infect Dis. 2018. https://doi.org/ 10.1016/S1473-3099(17)30628-X.

62. Wong MH, Shum H-P, Chen JH, Man M-Y, Wu A, Chan EW, et al. Emergence of carbapenem-resistant hypervirulent Klebsiella pneumoniae. Lancet Infect Dis. 2018. https://doi.org/10.1016/S1473-3099(17)30629-1.

Ready to submit your research? Choose BMC and benefit from:

- fast, convenient online submission

- thorough peer review by experienced researchers in your field

- rapid publication on acceptance

- support for research data, including large and complex data types

- gold Open Access which fosters wider collaboration and increased citations

- maximum visibility for your research: over $100 \mathrm{M}$ website views per year

At BMC, research is always in progress.

Learn more biomedcentral.com/submission 